\title{
O PRINCÍPIO DE PROJEÇÃO ESTENDIDA NO PORTUGUÊS BRASILEIRO*
}

\author{
Esmeralda Vailati Negrão**
}

\section{Introdução}

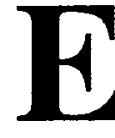

m Negrão (1999) defendi a tese de que o português brasileiro é uma língua que pode ser tipologicamente caracterizada como uma língua voltada para o discurso. Uma língua voltada para o discurso, foi tomada como sendo a língua que privilegia marcar na sintaxe aberta' a função informacional dos constituintes de sua sentença, ou seja, funções como tópico do discurso ou foco, ou ainda, o escopo ${ }^{2}$ de sintagmas quantificados. Tomando

* Os dados aqui apresentados foram extraf́dos de resultados parciais alcançados na pesquisa "Escopo dos sintagmas quantificados do português do Brasil", realizada com o apoio do CNPq, processo n.300654/97-9, a quem agradeço. Outras versões deste trabalho já foram apresentadas em encontros cientrficos no Brasil e no exterior, a cujos participantes agradeço os comentários recebidos.

** Universidade de São Paulo.

1 No jargão da Teoria Gerativa, sintaxe aberta significa, ou o nfvel de representaçăo conhecido como Estrutura-S no modelo de Princípios e Parâmetros, ou a ctapa da derivação antes da operação de Spell-out, que envia as informações para o componente fonológico, na visão minimalista desse modelo de gramática.

2 Ilari e Geraldi (1987, p. 33) definem escopo, no âmbito da semântica, como "conjunto de conteúdos semânticos sobre os quais uma operação significativa atua". $\mathrm{Na}$ 
NEGRĀO, E. V. O princípio de projeção estendida no...

a caracterização do nível de representação conhecido como Forma Lógica (LF) proposta por Hornstein (1995, p. 3):

(1) LF is the level of linguistic representation at which all grammatical structure relevant to semantic interpretation is provided,

podemos dizer que as sentenças das línguas voltadas para o discurso expressam visivelmente, ou seja , na forma como as sentenças são ouvidas, certas relações que outras línguas só vão marcar no nível de representação responsável por fornecer as estruturas que vão alimentar o componente interpretativo.

A caracterização de línguas voltadas para o discurso foi proposta por Huang $(1982,1984)$, face à constatação de que não só línguas com um paradigma de marcas morfológicas verbais capazes de identificar as pessoas do discurso admitem categorias vazias na posição de sujeito de suas sentenças, mas categorias vazias são também possíveis em línguas que não possuem um sistema de flexão verbal rico e que, portanto, se utilizam de outros mecanismos para a identificação do conteúdo dessas categorias.

O autor propõe, então, um parâmetro tipológico mais geral que dividiria as línguas do mundo em línguas orientadas para o discurso e línguas orientadas para a sentença. Dentre as propriedades caracterizadoras de uma língua voltada para o discurso está o fato de que o elemento proeminente ${ }^{3}$ em suas sentenças é o tópico ao passo que, nas línguas voltadas para a sentença, o elemento proeminente é o sujeito. Isso explica a presença de sujeitos expletivos nessas últimas línguas, uma vez que, pelo princípio de projeção estendida todas as sentenças precisam ter um sujeito. Chomsky (1981) propõe o Princípio de Projeção estabelecendo que os complementos de um núcleo lexical precisam ser realizados em todos os níveis de representação da gramática, isto é, estrutura-D, estrutura-S e forma lógica. Chomsky (1982) junta a esse princípio uma segunda cláusula, a de que todas as sentenças têm sujeito, e renomeia o princípio contendo as duas cláusulas, como o princípio de projeção estendida. No livro Knowledge of language (1986), ele dá uma motivação para o tratamento das duas propriedades pelo mesmo princípio, dizendo que a primeira cláusula trata da saturação das

Teoria Gerativa, escopo foi definido a partir da estrutura hierárquica dos constituintes da sentença. May (1985, p. 5) dá a seguinte definição:

(i) The scope of a is the set of nodes that a c-commands at LF;

(ii) a c-commands $b$ iff the first branching node dominating a dominates $b$ (and $a$ does not dominate $b$ ).

3 A expressão "elemento proeminente na sentença" vai ser entendida neste trabalho como o constituinte da sentença sobre o qual recai a predicação primária da sentença. 
NEGRÃO, E. V. O princípio de projeção estendida no...

exigências argumentais de um item lexical, a segunda diz respeito à saturação da exigência sintática de que um predicado precisa de um sujeito do qual ele predica.

Já nas línguas voltadas para o discurso, a estrutura sujeito-predicado ${ }^{4}$ não é básica, e as sentenças da forma tópico-comentário são muito numerosas, possivelmente não derivadas de outra estrutura mais básica.

\section{O português brasileiro (PB) como língua voltada para o discurso}

Dentre as propriedades caracterizadoras das línguas voltadas para o discurso apontadas por Huang, a característica exibida pelo português brasileiro que levou Negrão a incluí-la nesse parâmetro é o fato de que a predicação primária (noção usada para nomear a relação entre os dois constituintes maiores que formam uma sentença), não obrigatoriamente obedece o princípio de projeção estendida tal como comumente implementado na teoria gerativa, mas pode se estabelecer tanto entre um DP, ou QP, e os constituintes incluídos em projeções máximas de categorias funcionais como TP, como até mesmo projeções integrantes do sistema $\mathrm{CP}$, que, transformadas em predicado, às vezes pela atuação de algum operador, predicam desse $\mathrm{DP}$, ou QP, na posição de especificador de tal projeção. A motivação para esse tipo de movimento é a marcação da função informacional do constituinte sobre o qual recai a predicação. Essa característica explica porque em PB o sujeito da predicação ocupa uma posição fora da sentença, proposição praticamente consensual entre os estudiosos da sintaxe dessa língua.

Para Negrão, a motivação empírica para a caracterização do PB enquanto língua voltada para o discurso veio da realização do que talvez possamos chamar de um experimento, ${ }^{5}$ que mostrou que as estratégias de interpretação das categorias pronominais do PB são as mesmas, tanto com um sistema de flexão em que marcas flexionais oponham lps, 3ps, 1pp, 3pp, quanto com um sistema reduzidíssimo em que a única marca flexional é a de 3ps. Mesmo com um sistema de flexão "paupérrimo", instâncias de categorias vazias, tendo a elas associados traços $\mathrm{f}$ de $1 \mathrm{ps}, 3 \mathrm{ps}$ específico, $1 \mathrm{pp}, 3 \mathrm{pp}$, foram atestadas. A conclusão a que esse experimento nos leva é que pobreza de flexão não está diretamente

4 Mais adiante no trabalho vamos explicitar o que caracteriza uma estrutura sujeitopredicado por oposição à estrutura tópico-comentário.

5 Para uma descrição detalhada da execução desse experimento e das conclusōes dele advindas consultar Negrão (1990 e 1999). 
NEGRÃO, E. V. O princípio de projeção estendida no...

correlacionada a desaparecimento de categorias vazias, ou a realização de pronomes plenos. Portanto, as características observadas na gramática do português brasileiro não podem ser explicadas pelo enfraquecimento de seu sistema flexional, como fazem um conjunto de trabalhos que se debruçam sobre as mudanças em curso nessa língua. Mas, ao contrário, as propriedades que fazem do PB uma língua voltada para o discurso esvaziaram a função que o sistema de marcas flexionais tem nas línguas voltadas para o sujeito.

Dentre as evidências empíricas arroladas por Negrão(1999) para confirmar a inserção do PB no parâmetro de língua voltada para o discurso, quero aqui retomar $\mathbf{a}$ análise que visou a explicar a distribuição e $a$ interpretação de diferentes tipos de sintagmas quantificados. Partindo da proposta de que as propriedades de escopo dos sintagmas quantificados podem ser derivadas dos princípios reguladores da gramática das línguas naturais, as diferentes possibilidades de interpretação associadas aos diferentes tipos de sintagmas quantificados e a impossibilidade de algumas interpretações associadas a sintagmas quantificados atestadas em outras línguas tornam-se evidência para a postulação de uma estrutura sentencial característica do PB. Ao compararmos O PB ao inglês no que tange à distribuição e à interpretação de seus sintagmas quantificados, mais especificamente dos sintagmas distributivos quando interagindo com sintagmas denotadores de grupo e com sintagmas de contagem, observou-se que, no inglês, certas interpretações são geradas pelo movimento de tais sintagmas para as projeções funcionais associadas a sua interpretação em Forma Lógica, ao passo que, em PB, tal movimento é barrado pelo fato de o sujeito da predicação movimentar-se para a posição fora da sentença responsável por essa interpretação antes de Spell-out, o que explica a impossibilidade de certos padrões de escopo invertido nessa última língua. Desse modo, as diferenças observadas nos padrões de escopo das duas línguas pode ser atribuída a diferenças em suas estruturas sentenciais, corroborando a caracterização tipológica do PB como língua voltada para o discurso.

\section{A distribuição e a interpretação dos sintagmas quantificados do PB segundo a Teoria de Escopo de Aterrissagem Seletiva}

A interpretação associada a sentenças contendo Sintagmas Quantificados (QPS) ${ }^{6}$ é comumente derivada por mecanismos que dão ao QP a possibilidade de

6 Seguindo a tradição, neste texto as siglas são introduzidas entre parênteses logo 
ter escopo sobre o resto da sentença. Entendemos que um determinado QP tem escopo sobre outro QP quando a interpretação desse último depende da interpretação do primeiro.

Diferentes propostas foram feitas na literatura de tradição gerativista para implementar esses mecanismos. Uma dessas propostas foi desenvolvida por Beghelli (1995) e Beghelli e Stowell (1996). Reconhecendo que diferentes QPs comportam-se de maneira distinta com relação às possibilidades de escopo que podem assumir, os autores propõem uma tipologia de QPs para captar tais diferenças e desenvolvem uma teoria do escopo de quantificadores, que eles chamam de "Target landing site theory of scope" (Teoria de escopo de aterrissagem seletiva). Essa teoria: a) compartilha com as demais teorias sobre escopo de quantificadores dentro do modelo gerativo, da idéia de que a estrutura hierárquica geradora das relações sintáticas desempenha papel fundamental no estabelecimento das relações de escopo; b) distancia-se dessas teorias no momento em que rejeita o pressuposto de que as relações de escopo estabelecemse de maneira uniforme para todos os quantificadores. Os autores propõem que cada tipo de QP move-se em forma lógica(LF) para a posição de especificador (Spec) de uma projeção funcional específica a fim de checar o traço semântico que o caracteriza, estabelecendo uma relação de concordância com o operador ocupando o núcleo de tal projeção. Distinguem, assim, os seguintes tipos de QPs: QPs interrogativos (WhQPs), QPs negativos (NQPs), QPs distributivosuniversais (DQPs), QPs denotadores de grupo (GQPs) e QPs de contagem (CQPs). Essa tipologia, como eu disse, é baseada nas diferenças de escopo observadas quando esses QPs interagem com outros quantificadores ou operadores.

Como consequiência, essa teoria capta as diferenças empíricas atestadas na distribuição das leituras possíveis quando QPs interagem, por uma convergência de fatores: 1) os traços semânticos associados com cada tipo de QP checados na projeção funcional relevante; 2) a estrutura hierárquica de projeções funcionais: e 3) os princípios reguladores da teoria do movimento.

após a primeira aparição de sua forma desenvolvida. A partir de sua introdução, as siglas serão usadas sem sua contraparte desenvolvida, ao longo de todo o texto. Optei, também. por usar as siglas correspondentes às expressões $\mathrm{em}$ inglês. 


\section{A Sintaxe da distributividade e as assimetrias entre DQPs na posição de sujeito versus DQPs na posição de objeto do PB}

O fenômeno do escopo relativo advindo da interação entre sintagmas quantificados e/ou operadores numa dada sentença torna-se visivel através das possíveis interpretações associadas com as sentenças nas quais eles se inserem. Dentre os tipos de interpretação elucidativos das relações de escopo destaco: a interpretação distributiva e a interpretação de pressuposição de existência associada a QPs. Sentenças contendo sintagmas distributivos produzem a seguinte interpretação: "para cada membro, a, b, c,..., n, do conjunto introduzido pelo sintagma distributivo, existem eventos distintos, ou grupos distintos introduzidos por um outro QP, que estão associados com a, b, c,..., n, respectivamente". Assim, a uma sentença como:

(2) Cada aluno leu alguns textos.

que contém um sintagma distributivo interagindo com um sintagma denotador de grupo, posso atribuir a interpretação de que para pelo menos dois membros do conjunto denotado por aluno existem alguns textos que esse aluno leu, ou seja, a interpretação na qual alunos distribuem-se sobre grupos de alguns textos ou sobre eventos de leitura de alguns textos. Já em:

(3) Cada aluno leu os dois textos indicados para a aula.

a interpretação é de que existem dois textos indicados para a aula que cada aluno leu, ou seja, a leitura de pressuposição de existência de um grupo de dois textos indicados para a aula é evidência de que esse sintagma tem escopo largo sobre cada aluno que, por sua vez, distribui sobre eventos de leitura desses dois textos. Essa interpretação é gerada quando o sintagma ocupando a posição de objeto tem escopo largo sobre o sintagma ocupando a posição de sujeito e é chamada de "leitura de escopo invertido".

O problema interessante trazido por esses dados do $\mathrm{PB}$, uma língua voltada para o discurso, quando comparados aos dados de uma língua voltada para a sentença como o inglês, é que em PB sintagmas distributivos em posição de objeto podem causar a estranheza e até mesmo a agramaticalidade da sentença, como em:

(4) ?*Alguns alunos leram cada texto. 
Esse fato pode ser correlacionado a outro já observado para uma língua como o chinês por Aoun e Li (1993), qual seja, a impossibilidade de escopo invertido numa sentença do chinês correspondente a:

(5) Todo mundo prendeu uma mulher.

Em chinês, a interpretação de que existe uma única mulher que foi presa por todo mundo é impossível de ser associada à sentença (5), que só pode ser interpretada como representando a situação em que há uma mulher para cada elemento do grupo denotado por todo mundo. Por outro lado, as duas interpretações são possíveis para a versão inglesa dessa sentença. Aoun e Li derivam esse contraste nos padrões de escopo das duas línguas de diferenças nas respectivas estruturas sentenciais. Em inglês, o sintagma que acaba por ocupar a posição de SPEC- IP é gerado internamente a VP, enquanto que em chinês, SPEC-IP é a posição na qual sintagmas funcionando como sujeito de sentenças ativas são gerados. A mesma impossibilidade de escopo invertido na sentença (5) é observada no $\mathrm{PB}$.

A opção por adotar a teoria de Beghelli e Stowell em detrimento da de Aoun e Li para explicar a sintaxe das relações escopais de sentenças do PB advém do fato de que a última prediz a impossibilidade de interpretação de escopo invertido para seja qual for o tipo de sintagma quantificado ocupando a posição de objeto. Essa predição não se sustenta em PB. Em primeiro lugar, a agramaticalidade observada em (4) só se dá quando a posição de objeto á ocupada por sintagmas distributivos. O mesmo não ocorre com sintagmas denotadores de grupo ou sintagmas de contagem, nos termos de Beghelli e Stowell, como em:

(6) Alguns alunos leram dois textos.

A sentença (6) é uma sentença perfeita da língua, embora a leitura de escopo invertido lhe seja muito difícil, até mesmo impossível, de ser atribuida. Além disso, a sentença:

(7) Um repórter diferente entrevistou cada artista.

é plenamente aceitável, exatamente porque nesse caso o sintagma cada artista pode distribuir sobre repórteres, indicando a possibilidade de escopo invertido quando um QP distributivo interage com um QP de contagem.

A teoria proposta por Beghelli e Stowell dá conta de explicar esse conjunto de fatos postulando uma estrutura para a sentença em que uma série de projeções de categorias funcionais organizadas hierarquicamente oferecem sua 
NEGRÃO, E. V. O princípio de projeção estendida no...

posição de especificador como lugar de pouso para que cada um dos tipos de sintagmas quantificados possa mover-se para, numa relação de concordância, checar o traço semântico que lhe é peculiar. As leituras associadas a sentenças contendo QPS distributivos (DQPs) são geradas a partir da postulação de uma projeção funcional, o sintagma distributivo (DistP), tendo em seu núcleo Dist ${ }^{\circ}$ um operador distributivo, para cuja posição de especificador O DQP funcionando como distribuidor move-se, em LF, a fim de checar o seu traço semântico [ + distribuidor]. Dist. ${ }^{\circ}$ seleciona uma outra projeção funcional, o sintagma parte (Share $\mathrm{P}$ ), cuja posição de especificador vai ser ocupada, quer pelo quantificador existencial que prende o argumento evento associado com o predicado núcleo do sintagma verbal (VP), quer por outros tipos de sintagmas quantificados. Essa seleção capta a observação já feita por outros autores que estudaram a distributividade nas línguas naturais de que QPs distributivos necessitam de um domínio sobre o qual exerçam a distribuição. É por isso que os eventos ou grupos sobre os quais os DQPS distribuem receberam o nome de "share"(parte) e a projeção funcional que os abriga é chamada de "ShareP". Sendo assim, as possibilidades de escopo exibidas por QPs distributivos, isto é, a possibilidade de exibirem escopo estreito ou largo, não depende do movimento do DQP em si, uma vez que numa teoria de escopo que assume movimento seletivo de QPs em forma lógica para a posição de especificador de projeções funcionais específicas, a interpretação distributiva com eles associada decorre sempre do movimento desses QPS para a projeção funcional DistP. A possibilidade de escopo largo ou estreito depende sim da posição ocupada em LF pelos QPs com que os QPs distributivos interagem. A leitura em que cada-DQPs exibem escopo largo é obtida através de movimento do DQP para Spec de DistP e movimento do QP com que ele interage para uma posição dentro de seu domínio. Por outro lado, $c a d a$-DQPs exibe escopo estreito quando o QP com que eles interage move-se para uma posição superior, isto é, fora de seu domínio.

Usando a teoria de Beghelli e Stowell, Negrão (1999) consegue explicar não só a agramaticalidade da sentença (4), como também a ausência de escopo invertido numa sentença como (5), correlacionando ambos os fenômenos ao fato de que em PB, sentenças em que a predicação primária se dá entre um sujeito, cuja existência é pressuposta, e um predicado que, em termos estruturais, corresponde a todo o complexo TP já exibem essa representação antes de Spellout, diferentemente de outras línguas em que essa representação só é obtida em LF. A sentença correspondente a (4) em inglês não só é gramatical, como também é ambígua, tendo a ela associada tanto a interpretação em que alguns alunos têm escopo largo sobre cada texto (ver gráfico 8), quanto a interpretação em que cada texto tem escopo largo sobre alguns alunos (ver gráfico 9). 
(8)

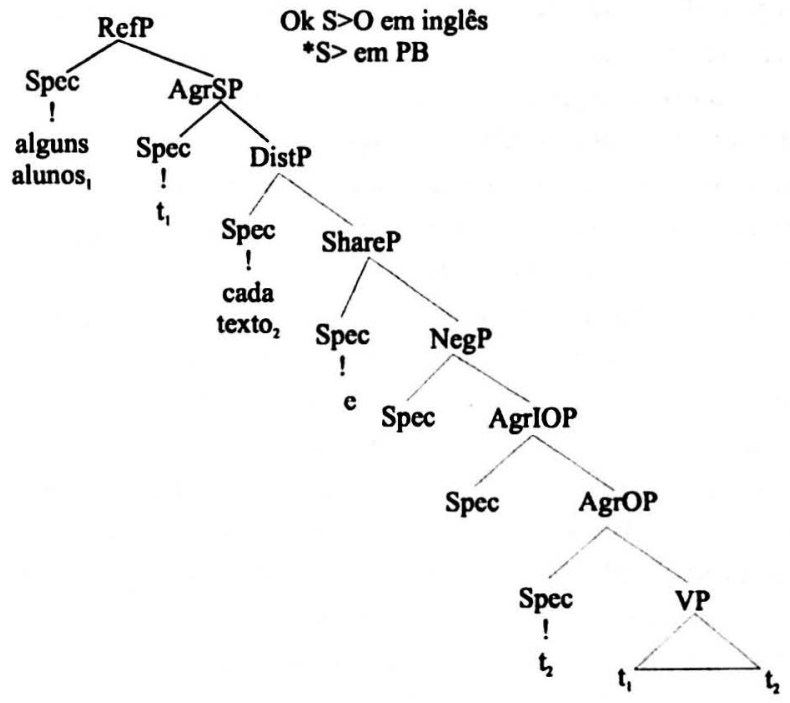

(9)

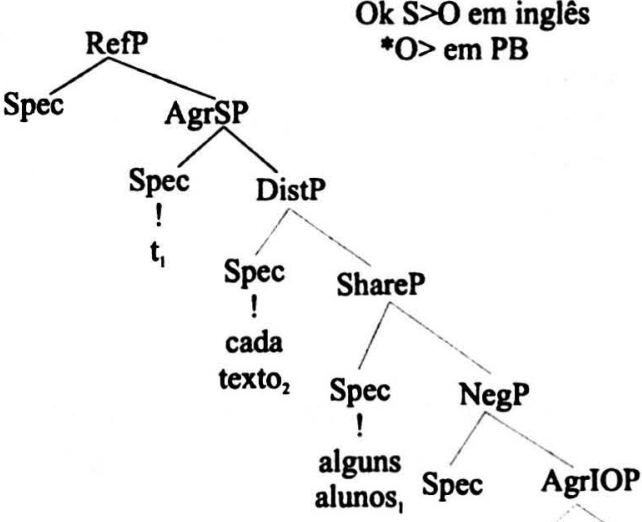

Spec

AgrOP

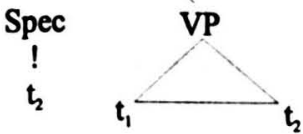


A impossibilidade das duas derivações em PB pode ser explicada da seguinte maneira. Dadas as fortes exigências de distributividade de cada, só serão possíveis as sentenças em que ele tenha sob seu escopo, ou um sintagma que lhe ofereça um grupo, ou uma quantificação sobre eventos que lhe ofereça eventos sucessivos, aos quais possa associar os elementos do grupo denotado por sua restrição. Na representação em (8), o fato de o sintagma alguns alunos ter escopo largo, tira a possibilidade de ele servir como domínio de distribuição. A outra alternativa seria a quantificação sobre eventos que, por nesse caso expressar a existência de um único evento, também não satisfaz as exigências distributivas de cada. $\mathrm{Na}$ representação em (9), por sua vez, as exigências distributivas de cada são satisfeitas pelo fato de o sintagma alguns alunos, sofrendo um processo que Beghelli trata como de reconstrução na posição de especificador de ShareP, terminar em LF sob o escopo de cada texto. Se (9) é uma forma lógica impossível para a sentença (4) do $\mathrm{PB}$, é porque alguns alunos não pode terminar sob o escopo de cada texto. Negrão deriva essa impossibilidade do fato de que em PB, sintagmas denotadores de indivíduos e de grupos, que desempenharão a função de sujeito da predicação, movem-se para a posição que Beghelli e Stowell chamam de RefP, antes de Spell-out, e por isso não podem sofrer reconstrução. Desse modo, as diferenças observadas nos padrões de escopo das duas línguas pode ser atribuída a diferenças em suas estruturas sentenciais.

Embora seja inegável que a postulação de um conjunto de projeções funcionais é indesejável, do ponto de vista teórico, pelo alto poder descritivo que elas conferem à teoria gramatical, a teoria proposta por Beghelli e Stowell possibilitou a descrição e a explicação de um conjunto de fatos do PB que não mais poderão ser deixados de lado por propostas de soluções teóricas mais econômicas.

\section{Tentativa de tratamento das generalizações descritivas alcançadas numa estrutura sentencial mínima}

A análise proposta na seção anterior traz à tona a preocupação com a tensão subjacente à pesquisa em teoria gerativa entre a adequação descritiva $\mathbf{e}$ a adequação explicativa. Diz Chomsky (1995, p. 317): "These efforts were motivated by the usual dual concerns: the empirical demands posed by the problems of descriptive and explanatory adequacy, and the conceptual demands of simplicity and naturalness." 
O programa minimalista de investigação do conhecimento lingüístico humano coloca-se o objetivo de resolução dessa tensão. Sendo assim, não basta para justificar a postulação de uma categoria, o fato de ela dar conta da descrição de um conjunto de fatos empíricos. O padrão de organização dos constituintes responsáveis pela estruturação das sentenças é reformulado no sentido de que dele sejam eliminadas as categorias que não gramaticalizem aspectos da significação, como por exemplo, a categoria concordância (AGR). A sentença numa gramática mínima passa a ter a seguinte estrutura:

\section{(10) $\left\{_{\mathrm{CP}}\left[{ }_{\mathrm{TP}}\left[{ }_{\mathrm{vP}}\left({ }_{\mathrm{VP}}\right)\right]\right]\right\}$}

A seqüência de dois VPs é justificada pela necessidade de realização da estrutura argumental dos verbos, captando as assimetrias hierárquicas entre os argumentos. O sintagma temporal é justificado porque carrega as marcas de tempo do evento descrito pela sentença e o sintagma complementizador porque abriga o traço referente à operação de interrogação. A questão agora é como, nesse modelo mínimo, podemos dar conta dos fenômenos analisados na seção anterior. Antes de mais nada é preciso ressaltar que, nesse modelo de gramática, movimentos de constituintes atendem a necessidades morfológicas. Sendo assim, para mover-se, o constituinte precisa ser atraído por uma categoria que, por apresentar um determinado traço, exige que o constituinte portador de um traço compatível mova-se para a posição de especificador de sua respectiva projeção.

Chomsky (1995) esboça os principais pontos no tratamento dos padrões de escopo de sintagmas quantificados numa gramática minimalista. Assume a operação conhecida como "alçamento de quantificador", diz que por ser um movimento coberto é um movimento do traço [quant], um traço interpretável e que, portanto, não precisa ser checado. $O$ traço sobe adjungindo-se a um $X^{0 \max }$, talvez $\mathrm{T}$ ou $\mathrm{v}$, que por sua vez tem traços opcionais de afixo que permitem abrigar [quant]. Ser opcional significa que esse traço só é escolhido se ele tiver conseqüências no resultado final da derivação, sendo portanto motivado por princípios de economia.

Fox (2000) defende a relevância dos princípios de economia na determinação da estruturação da sentença que alimenta o componente semântico e implementa o tratamento minimalista às sentenças envolvendo sintagmas quantificados. Utilizando-se de evidências vindas da interpretação de construções com elipse, mostra o papel que os princípios de economia desempenham na determinação dos padrões de escopo. Propõe, então, o seguinte princípio: 
NEGRÃO, E. V. O princípio de projeção estendida no...

(11) Scope Economy (Fox, 2000, p. 3)

Scope-shifiting operations (SSO) cannot be semantically vacuouos,

ou seja, a operação de alçamento de quantificador só se aplica, quando necessária para derivar uma dada interpretação semântica. Por meio dessa operação, o constituinte que tem escopo largo é adjungido, cobertamente, a uma categoria funcional. As sentenças em (12), e suas respectivas formas lógicas - (13) expressando a interpretação sem escopo invertido e (14), a interpretação com escopo invertido - que reproduzem os exemplos (2), (6) e (7) de Fox (2000, p. 34), exemplificam a proposta:

(12) a. A boy loves every girl . um menino ama toda garota.

b. Many boys love every girl. muito meninos amam toda garota.

(13)a. $\left\{\left\{_{I P}\right.\right.$ a boy $\ldots\left[_{V P}\right.$ every girl ${ }_{2}\left({ }_{V P} t_{1}\right.$ loves $\left.\left.\left.t_{2}\right)\right]\right\}$

b. $\left\{\right.$ many boys ${ }_{1} \ldots\left[\left[_{V P}\right.\right.$ every girl ${ }_{2}\left(\mathrm{v}_{\mathrm{VP}} \mathrm{t}_{1}\right.$ love $\left.\left.\left.\mathrm{t}_{2}\right)\right]\right\}$

(14)a. $\left\{\begin{array}{l}\text { IP } \\ \text { every girl }{ }_{2}\left[{ }_{I P} \text { a boy }\right. \\ 1\end{array}\left[_{V P} t_{2}\left({ }_{V P} t_{1}\right.\right.\right.$ loves $\left.\left.\left.\left.t_{2}\right)\right]\right]\right\}$

b. $\left[{ }_{I P}\right.$ every girl ${ }_{2}\left[\right.$ many boys $_{1} \ldots\left[_{V P} t_{2}\left[{ }_{V P} t_{1}\right.\right.$ love $\left.\left.\left.\left.t_{2}\right]\right]\right]\right]$

Como, então, tratar as generalizações descritivas alcançadas na análise do escopo dos sintagmas quantificados, proposta na sessão 3, numa estrutura sentencial mínima? Tal como formuladas, as propostas de Chomsky e Fox, resumidas acima, não podem dar conta da heterogeniedade dos sintagmas quantificados. Delineio, a seguir, um esboço de tratamento que tenta contemplar tal heterogeneidade numa gramática minimalista.

Comecemos por, com Chomsky (1998, p. 35), assumir que: "The EPPfeature of $\mathrm{T}$ might be universal. For the phase heads $\mathrm{v} / \mathrm{C}$, it varies parametrically among languages and if available is optional."

Suponhamos que os diferentes tipos de quantificadores que introduzem sintagmas quantificados tragam os traços interpretáveis: [dist] se for um DQP, [group] se for um GQP e [count] se for um CQP. Por ser uma língua voltada para o discurso, o $P B$ pode realizar o EPP em CP: $C P$ tem um traço $D$ forte que pode ser checado, ou por DQPs, ou por GQPs. Já TP tem somente um traço nominal forte que é checado por CQPs, sintagmas quantificados que não pressupõem a existência do grupo denotado por sua restrição. 


\section{Considerações Finais}

A proposta sugerida parece que consegue dar conta das generalizaçōes empíricas alcançadas na análise do escopo dos sintagmas quantificados, por meio de uma solução mais econômica. Nesse sentido, torna-se um projeto bastante interessante desenvolvê-la por completo, na medida em que ela alia o alto poder descritivo da análise realizada ao forte poder explicativo da concep̧̧ão minimalista de gramática.

\section{RESUMO}

Neste trabalho, parto da tese de que o português brasileiro (PB)é uma língua que pode ser tipologicamente caracterizada como língua voltada para o discurso, ou seja, uma língua que privilegia marcar, na sintaxe aberta, a função informacional dos constituintes de sua sentença, ou seja, funções como tópico do discurso ou foco, ou ainda, o escopo de sintagmas quantificados. A impossibilidade da interpretação de escopo invertido de certas sentenças do PB é tomada como evidência de que a predicação primária (noção que está sendo usada para nomear a relação entre os dois constituintes maiores que formam uma sentença), não obrigatoriamente obedece o princípio de projeção estendida (EPP) tal como proposto pela teoria gerativa no modelo de princípios e parâmetros, mas pode se estabelecer entre um DP, ou QP, e os constituintes incluídos em projeçōes máximas de categorias funcionais como TP e até mesmo projeções integrantes do sistema CP, que, transformadas em predicado, às vezes pela atuação de algum operador, predicam desse DP, ou QP, na posição de especificador de tal projeção. Em trabalho recente, Chomsky (1998) abre a possibilidade de as línguas variarem com relação à satisfação do EPP, agora tratado como um traço de categorias funcionais, embora ainda considere o traço EPP de T como universal. Investigo, então, as conseqüências dessa abertura para o tratamento das sentenças do PB contendo sintagmas quantificados, para que os seus padrōes de escopo possam ser derivados por meio de uma solução mais econômica.

Palavras-chave: Teoria gerativa, escopo de sintagmas quantificados, forma lógica, categorias funcionais. 
NEGRÃO, E. V. O princípio de projeção estendida no...

\section{ABSTRACT}

In this paper, I assume the thesis that Brazilian Portuguese (BP) is a discourseoriented language, in the sense that it makes visible in overt syntax the informational function of certain constituents, such as, discourse topic and focus, and the scope of quantifier phrases (QPs). The impossibility of an inverse scope reading associated with sentences containing QPs is taken as evidence for the claim that, in such languages, the basic predicative relation is not one established between the subject and the predicate within IP, as expressed in the Principles \& Parameters formulation of the Extended Projection Principle (EPP), but it is established between the whole IP and a constituent included in a functional projection of the CP system. In recent work, Chomsky (1998) opens the possibility for language variation in regard to the EPP satisfaction. I then investigate the consequences of this new approach to the treatment of sentences containing QPS in order to derive scope patterns within a more economical framework.

Key-words: Generative grammar, scope of quantifier phrases, logical form.

\section{REFERÊNCIAS}

AOUN, J.; LI, Y. A. (1993). Syntax of scope. Cambridge, Mass.: MIT Press.

BEGHELLI, F. The phrase structure of quantifier scope. Los Angeles, 1995. Dissertation (Ph. D.) - University of California.

BEGHELLI, F.; STOWELL, T. (1997). Distributivity and negation: the syntax of $E A C H$ and EVERY. In: SZABOLCSI, A. (Ed.) Ways of scope taking. Dordrecht: Kluwer. p. 71 109.

CHOMSKY, N. (1981). Lectures on government and binding. Dordrecht: Foris.

CHOMSKY, N. (1982). Some concepts and consequences of the theory of government and binding. Cambridge, Mass.: MIT Press.

CHOMSKY, N. (1986). Knowledge of language. New York: Praeger.

CHOMSKY, N. (1996). The minimalist program. Cambridge, Mass.: MIT Press.

CHOMSKY, N. (1998). Minimalist inquires: the framework. (mimeo.) 
FOX, D. (2000). Economy and semantic interpretation. Cambridge, Mass.: MIT Press. HORNSTEIN, N. (1995). Logical Form: from GB to Minimalism. Cambridge, Mass.: Blackwell.

HUANG, C.-T. J. Logical relations in Chinese and the theory of grammar. Cambridge Mass, [19-]. Dissertation (Ph. D.) - MIT.

HUANG, C.-T. J. (1984). On the distribution and reference of empty pronouns. Linguistic Inquiry, v. 15, n. 4, p. 531-574.

ILARI, R.; GERALDI, J. W. (1987). Semântica. São Paulo: Ática. (Série Princípios).

MAY, R. (1985). Logical Form. Its structure and derivation. Cambridge, Mass.: MIT Press.

NEGRÃO, E. V. (1990). A distribuição e a interpretação de pronomes na fala de crianças da escola pública. São Paulo: Relatónio de pesquisa financiada pela Fapesp. (Mimeog.).

NEGRÃO, E.V. (1999). O português brasileiro: uma língua voltada para o discurso. São Paulo, 1999. Tese (Livre-Docência) - Universidade de São Paulo. 